\title{
Enhanced Electroluminescence of Polymer Light-Emitting Diodes with Direct Polyaniline Synthesized Anodes
}

\author{
Chii-Chang Chen, ${ }^{\dagger}$ S.R. Hwang, Wen-Hsien LI, Kuan-Ching LeE, Gou-Chung CHI, \\ Hsia-Tsai HsiaO, ${ }^{*}$ and Chun-Guey Wu* \\ Department of Physics, National Central University, Chung-Li 32054, Taiwan, Republic of China \\ ${ }^{*}$ Department of Chemistry, National Central University, Chung-Li 32054, Taiwan, Republic of China
}

(Received November 27, 2001; Accepted February 23, 2002)

\begin{abstract}
We report the current-voltage and the electroluminescence characteristics of the light-emitting diodes based on poly[2-methoxy-5-(2'-ethylhexyloxy)-1,4-phenylenevinylene] (MEH-PPV) with indium-tin oxide (ITO) anodes and the diodes in which a layer of polyaniline (PANI) is synthesized directly on a layer of self-assembled monolayers formed by $N$-phenylaminopropyltrimethoxysilane above the ITO. The results show that the PANI layer reduces Schottky energy barrier and enhances the electroluminescence intensity. We believe that the insertion of the PANI layer causes dipole layers established on the surface resulting in the reduction of the energy barrier. The PANI layer also affects the electron-hole transition probability of the active layer enhancing the brightness of the devices dramatically.

KEY WORDS Polymer Light-Enitting Diode (PLED) / Indium-Tin Oxide (ITO) / Polyaniline (PANI) /
\end{abstract}

Since the discovery of electroluminescence in polymers, ${ }^{1}$ their external power efficiency, color versatility, brightness, angular emission profile and weight are all attractive for use in flat-panel and portable displays. In addition, the wavelength of emission can be tuned by reducing the conjugation length in the polymer backbone. $^{2}$ The quantum efficiency and the driving voltage of the devices depend on the charge-injection properties at the interfaces between the electroluminescent polymer and electrodes. Recently, the chain scission of the electroluminescent polymer has been enhanced by the chemical reaction of the vinyl carbon with oxygen contributed by the indium-tin oxide (ITO) anode. ${ }^{3}$ These results have shown that the ITO/polymer interface plays an important role in device lifetime.

To improve the brightness and the lifetime of the polymer light-emitting diodes (PLEDs), the insertion of the polyaniline (PANI) anode was intensively studied. ${ }^{3-6}$ The barrier height at the PANI/MEH-PPV interface has been reduced to $0.08-0.12 \mathrm{eV}$ that is roughly half of the at the ITO/MEH-PPV interface. This results in decreasing the device operating voltage and increasing the quantum efficiency. The effect due to the insertion of the PANI anode also showed the enhancement of the device lifetime that attributes to the reduction of the rate of oxidative degradation of the active layer. In this work, we have synthesized the PANI layer directly on the ITO substrate from the aniline monomer. ${ }^{7}$ The reason for the brightness enhancement and the increase of the tunneling current due to the PANI coated anode were discussed.

\section{EXPERIMENTAL}

Figure 1 shows the cross section of our samples. The ITO glass was cut into $2 \times 2 \mathrm{~cm}^{2}$ and was cleaned by potassium-tert-butylate, acetone, deionized water, and methyl alcohol sequentially in an ultrasonic bath for $30 \mathrm{~min}$. The ITO glass was immersed in the acidic solution which was prepared by mixing the dilute sulfuric acid $(0.36 \mathrm{M})$ and hydrogen peroxide in $3: 7$ volume ratio for 40 min to produce $\mathrm{OH}^{-}$on the ITO glass surface. The ITO glass was dipped into the methyl alcohol mixed with $N$-phenylaminopropyltrimethoxysilane inside a nitrogen filled glove box for $14 \mathrm{~h}$. After this procedure, a monolayer aniline-contaning silane compound was formed on the ITO substrate. A hy-

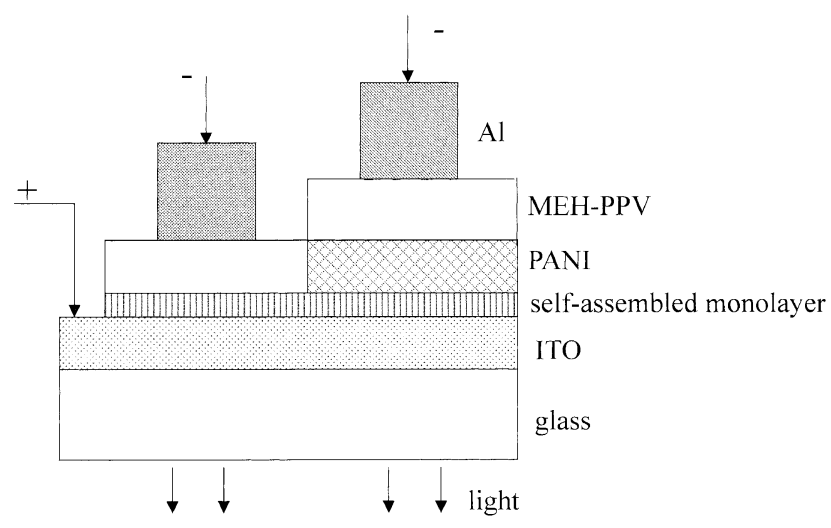

Figure 1. The cross section of the PLED device. A $500 \AA$ thick PANI layer was coated on half of the diodes surface.

${ }^{\dagger}$ To whom correspondence should be addressed (Tel: +886-3-4227151 ext. 5343, Fax: +886-3-4258816, E-mail: chx@phy.ncu.edu.tw). 
drochloric acid solution complex with ammonium peroxodisulfate was prepared in separate flasks. The ITO glass was dipped into the aniline solution mixed with the hydrochloric acid first and the ammonium peroxodisulfate solution was added. The reaction was performed in an ice bath for $17 \mathrm{~min}$. During the reaction, the aniline was oxidatively polymerized on the ITO substrate, and the color of the solution changed from transparent to green and then blue. After the PANI coated ITO glass was rinsed with deionized water, half the surface of the PANI coated ITO glass was dipped into the hydrochloric acid solution $(1.2 \mathrm{M})$ until the ITO glass changed color from purple to green. A thin layer of conducting polymer doped with hydrochloric acid was synthesized on the ITO glass. The synthesis of poly[2-methoxy-5-(2'-ethylhexyloxy)-1,4phenylenevinylene] (MEH-PPV) followed the reported procedure in ref 8 . The MEH-PPV $(6 \mathrm{mg})$ was dissolved in toluene $(1 \mathrm{~mL})$ and spin-coated on the ITO glass. A $2000 \AA$-thick Al thin film was deposited on the samples by thermal evaporation deposition with a shadow mask to produce a $1 \mathrm{~mm}^{2}$ diode area. Each sample consisted of the ITO/PANI+HCl/MEH-PPV/Al (PANI diode) and the ITO/MEH-PPV/Al (diode without PANI layer) as shown in Figure 1. After the fabrication process, the current was injected into the device to measure the electroluminescence spectra. The light emitted from the device is focalized by lenses into a 0.55 -m monochromator and analyzed by a photomultiplier tube.

\section{RESULTS AND DISCUSSION}

Figure 2 shows the current-voltage measurement at the ambient environment for the PANI diode and the diode without PANI layer. The tunneling voltage for both of the diodes are about 7 volts. The current for the PANI diode increased dramatically for the voltages beyond 7 volts. This indicates that under the same driving current, the consumed power of the PANI diodes is less than the diodes without PANI layer. The enhancement of the brightness can be observed in the electroluminescence spectra of the diodes at $20 \mathrm{~mA}$ (Figure 3). The integrated intensity is about nine times larger for the PANI diodes than the diodes without PANI layer.

In order to investigate the effects of the selfassembled monolayer at the ITO surface, we measured the electroluminescence spectra for two samples with ITO/MEH-PPV/Al structure (Figure 4). One sample followed the modification process as described above. The other sample is fabricated with the ITO as received. The spectra showed no obvious intensity difference indicating that the self-assembled monolayer on the ITO

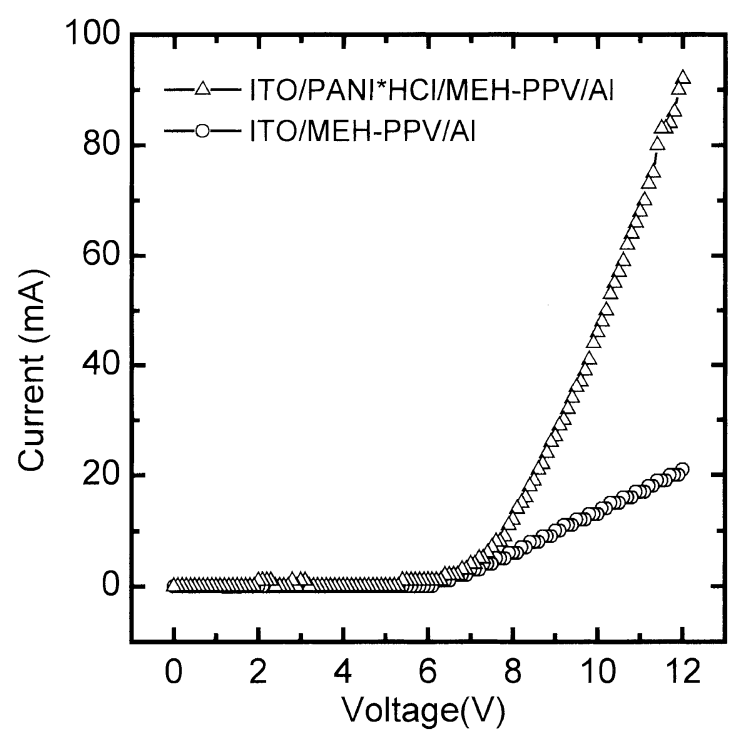

Figure 2. The $I-V$ characteristics of the diodes under the ambient environment. The diode with PANI anode shows a larger tunneling current.

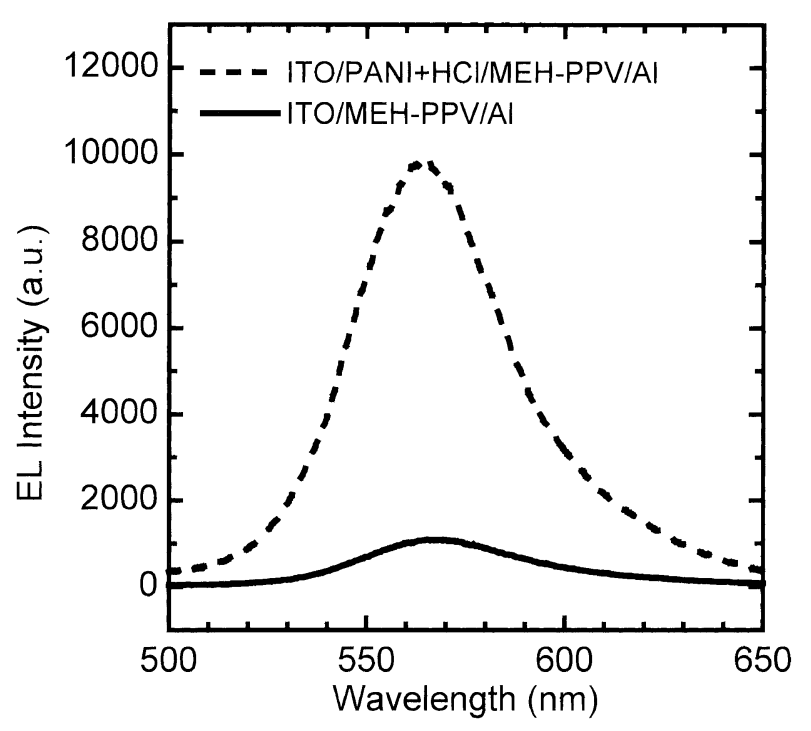

Figure 3. The electroluminescence spectra for the diodes with a PANI anode and without PANI anode, respectively.

layer does not contribute to the enhancement of the brightness in the PANI diodes. Therefore, the electroluminescence intensity enhancement should be dominated by the PANI layer.

We have estimated the barrier heights from the I-V measurement shown in Figure 2 based on the FowlerNordheim field-emission tunneling theory: ${ }^{9}$

$$
I \propto E^{2} \exp \left(\frac{-\kappa}{E}\right)
$$

where $I$ is the current, $E$ is the electric field strength, and $\kappa$ is the parameter that depends on the potential profile of the barrier at the metal-polymer contact. Assuming that the injected charges tunnel through a triangular barrier at the interface, the constant $\kappa$ in eq 1 is given 


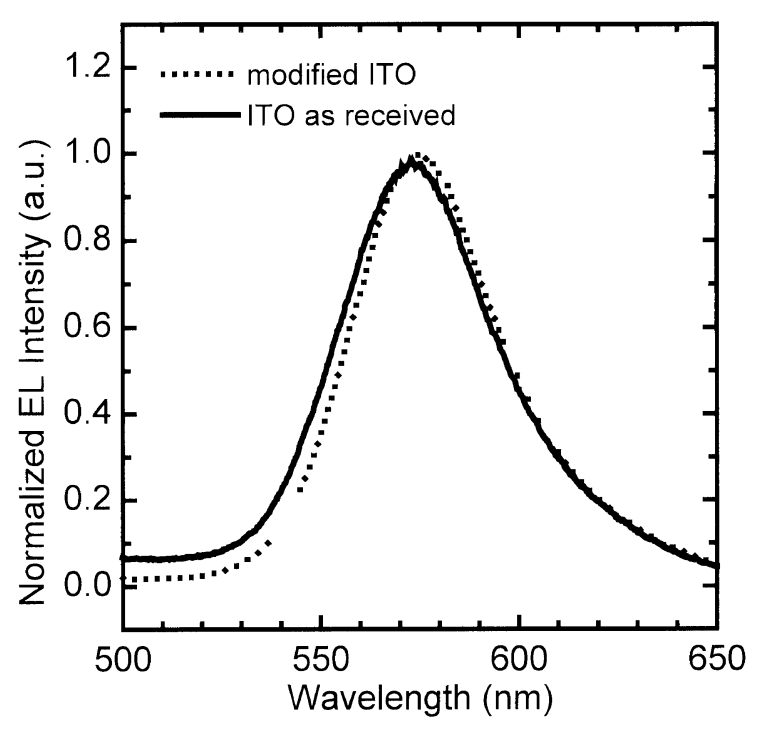

Figure 4. The electroluminescence spectra of the diode with a modified ITO and the diode fabricated with an ITO as received.

by ${ }^{10}$

$$
\kappa=\frac{8 \pi \sqrt{2 m^{*}} \phi^{3 / 2}}{3 q h}
$$

where $\phi$ is the barrier height and $m^{*}$ is the effective mass of the holes in the active layer (MEH-PPV). $q$ and $h$ are the elementary charge and Plank constant, respectively. We have assumed that the electric field is constant across the device and that the effective mass of the holes is equal to the free electron mass. Based on these assumptions, the theoretical prediction for the MEMPPV devices with electrodes having a wide range of work functions has shown an agreement with the selfconsistent experiments. ${ }^{11}$ Figure 5 shows a FowlerNordheim relation between $\ln \left(I / E^{2}\right)$ and $1 / E$ for the PANI diode and the diode without PANI layer. As predicted, the relation is linear under high electric fields, i.e., above the operating-voltage threshold. The deviation from linearity under lower electric fields is partly due to bulk conduction through the polymer. This effect might be comparable to or greater than the tunneling contribution. ${ }^{12}$ In the linear region, the calculated barrier heights for hole tunneling into the devices are $0.12 \mathrm{eV}$ for the PANI diodes and $0.20 \mathrm{eV}$ for the diodes without PANI layer, respectively. This indicates that the hole tunneling does not occur at the cathode due to the fact that the difference of the work function is over $1 \mathrm{eV}$ for the cathode (for example, the band offset values for $\mathrm{MEH}-\mathrm{PPV} / \mathrm{Al}$ and MEH-PPV/Ag are $1.2-1.6 \mathrm{eV}$ and $1.5-1.9 \mathrm{eV}$ respectively. $\left.{ }^{11}\right)$. The barrier heights derived from Figure 2 are consistent with the results in ref 5. The result reveals that the tunneling occurs across the ITO/polymer interface. This explains that the tunneling current can be enhanced in the PANI diode. The mechanism contributes to this barrier height reduction

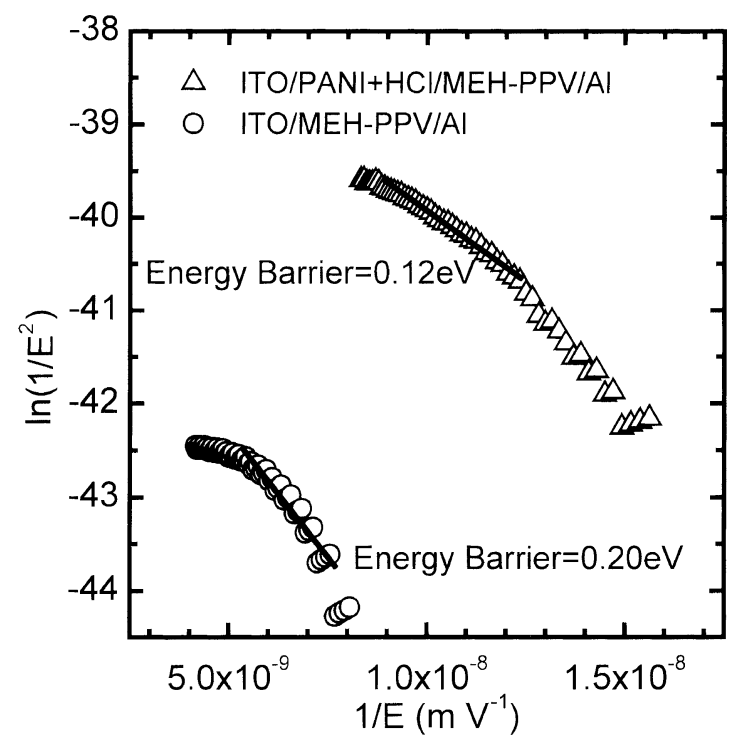

Figure 5. The Fowler-Nordheim plot for the energy barrier height calculations of the PANI diode and the diode without PANI layer.

might be related to dipole layers formed on the PANI layer. The barrier height can be tuned over a range of more than $1 \mathrm{eV}$ by attaching well-defined dipole layer to metal contacts prior to fabrication of the device. ${ }^{13} \mathrm{~A}$ $3-n m$ blue shift is observed in the electroluminescence spectra (Figure 3) after a PANI layer was inserted into the structure of the diode. This indicates that the energy band gap of the active layer was enlarged due to the PANI layer insertion. This phenomenon might attribute to the reduction of the Stark shift due to the fact that the additional electric field of the well-defined dipole compensates the bending of the triangular barrier at the interface. This effect can also increase the electron-hole transition probability leading to the enhancement of the device efficiency.

\section{CONCLUSION}

We have synthesized PANI directly on the modified ITO substrate with a self-assemble monolayer. The PANI diodes show a stronger brightness, a lower operation voltage, a larger tunneling current, a longer lifetime, and an operation stability than the diodes without PANI layer. The results confirm that the tunneling current occurs across the ITO/polymer interface. The reduction of the ITO/PANI barrier height might be due to the dipole layer formation mechanism on the PANI layer. The reduction of the Stark shift effect due to the PANI layer insertion might provide a higher electron-hole transition probability. These effects improve the tunneling current and the brightness for the PANI diodes. 


\section{C.-C. CHen et al.}

Acknowledgments. The authors would like to thank the useful help from Mr. C. H. Kuo and Ms. Lin$\mathrm{Yu}$ Chien for the sample preparation and the measurements.

\section{REFERENCES}

1. J. H. Burroughes, D. D. C. Bradley, A. R. Brown, R. N. Marks, K. Mackey, R. H. Friend, P. K. Burns, and A. B. Holmes, Nature, 347, 29 (1990).

2. C. Zhang, D. Braun, and A. J. Heeger, J. Appl. Phys., 73, 5177 (1993).

3. J. C. Scott, J. Kaufman, P. J. Brock, R. DiPietro, J. Salem, and J. A. Goitia, J. Appl. Phys., 79, 2745 (1996).

4. Y. Yang, E. Westerweeie, C. Zhang, P. Smith, and A. J. Heeger, J. Appl. Phys., 77, 694 (1995).
5. Y. Yang and A. J. Heeger, Appl. Phys. Lett., 64, 1245 (1994).

6. J. C. Scott, S. A. Carter, S. Karg, and M. Angelopoulos, Synth. Met., 85, 1197 (1997).

7. Y. R. Yeh, Master thesis in Department of Chemistry, National Central University, 2000.

8. C. J. Neef and J. P. Ferraris, Macromolecules, 30, 8094 (2000).

9. R. H. Fowler and L. Nordheim, Proc. R. Soc. London, Ser. A, 119, 173 (1958).

10. S. M. Sze, "Physics of Semiconductor Devices", John Wiley \& Sons, Inc., New York, N.Y., 1981, p 497.

11. I. D. Parker, J. Appl. Phys., 75, 1656 (1994).

12. P. S. Davids, I. H. Campbell, and D. L. Smith, J. Appl. Phys., 82, 6319 (1997).

13. I. H. Campbell, S. Rubin, T. A. Zawodzinski, J. D. Kress, R. L. Martin, D. L. Smith, N. N. Barashkov, and J. P. Ferraris, Phys. Rev. B, 54, 14321 (1996). 